\title{
Optical Response of Magnetic and Piezoelectric Materials to External Fields*
}

\author{
Shohei Abe \\ Department of Environmental System Engineering, Akita National College of Technology, \\ 1-1 Iijima-bunkyo-cho, Akita, Akita 011-8511, Japan \\ Koh-ichi Maruyama ${ }^{\dagger}$ \\ Department of Applied Chemistry, Akita National College of Technology, \\ 1-1 Iijima-bunkyo-cho, Akita, Akita 011-8511, Japan \\ Fumito Sakamoto \\ Department of Electric Engineering 8 Information Technology, \\ Akita National College of Technology, 1-1 Iijima-bunkyo-cho, Akita, Akita 011-8511, Japan \\ Hitoshi Yamaguchi \\ Center for Strategic Natural Resources, National Institute for Materials Science, \\ 1-2-1 Sengen, Tsukuba, Ibaraki 305-0047, Japan \\ Masato Yasumoto \\ National Institute of Advanced Industrial Science and Technology, \\ 1-1-1 Umezono, Tsukuba, Ibaraki 305-8568 Japan \\ Keiko Abe \\ Tech. Cent., Ichinoseki National College of Technology, \\ Takanashi, Hagiso, Ichinoseki, Iwate 021-8511, Japan \\ Yuki Sasaki and Akari Sugawara \\ Department of Applied Chemistry, Akita National College of Technology, \\ 1-1 Iijima-bunkyo-cho, Akita, Akita 011-8511, Japan. \\ (Received 27 January 2012; Accepted 30 August 2012; Published 15 September 2012)
}

\begin{abstract}
Optical measurement systems were constructed to investigate the magneto-optical (MO) effect and the piezoelectric-optical (PO) effect. The reflection PO effect of a lithium niobate $\left(\mathrm{LiNbO}_{3}\right)$ single crystal was affected by the inverse piezoelectric effect. The inverse piezoelectric effect was reduced in a sputtered $\mathrm{LiNbO}_{3}$ film. The signal intensity of the PO effect of a $\mathrm{LiNbO}_{3}$ film was of the same order $(0.01 \%)$ as that of the transverse $\mathrm{MO}$ Kerr effect of a spin-coated nickel ferrite $\left(\mathrm{NiFe}_{2} \mathrm{O}_{4}\right)$ film.
\end{abstract}

[DOI: $10.1380 /$ ejssnt.2012.503]

Keywords: Laser methods; Dielectric phenomena; Piezoelectric effect; Insulating surfaces; Semiconducting surfaces

\section{INTRODUCTION}

Multiferroic materials are promising for use in future electrical and spin devices [1] since they exhibit both ferromagnetic and ferroelectric properties. We have examined a hybrid material that consists of two layers: a ferromagnetic (or superparamagnetic) layer and a piezoelectric layer (Fig. 1). Applying an electric field to this material generates stress at the interface between the two layers due to dielectric strain in the piezoelectric phase. In addition, interfacial stress produces magnetostriction, which results in magnetization reversal and a magnetic phase transition; this is the inverse magnetostriction effect. Applying an electric field by irradiating this hybrid material with polarized light gives rise to two optical responses. One is the piezoelectric-optical (PO) effect. It involves a change in the dielectric constant due to strain

${ }^{*}$ This paper was presented at the 6th International Symposium on Surface Science - Towards Nano, Bio and Green Innovation-, Tower Hall Funabori, Tokyo, Japan, December 11-15, 2011.

$\dagger$ Corresponding author: maruko@akita-nct.jp (the electric-optical effect) and due to a change in the sample length (the inverse piezoelectric effect). The other optical response is the magneto-optical (MO) effect. This is the interaction between the magnetization of a magnetic material and the electric field of the electromagnetic wave. It is thus necessary to separate these effects (i.e., the piezoelectric-optical and magneto-optical effects [2]) to clarify the properties of the hybrid material (i.e., the spin and the electric polarization).

This study focuses on the optical responses of the magnetic and dielectric phases of a hybrid material. The purposes of this study are: (1) to construct an optical measurement system for detecting the MO and PO effects; (2) to observe the MO transverse Kerr effect in sol-gel $\mathrm{NiFe}_{2} \mathrm{O}_{4} / / \mathrm{Si}(100) ;(3)$ to observe the transmission and reflection $\mathrm{PO}$ effects in a $\mathrm{LiNbO}_{3}$ single crystal and a $\mathrm{LiNbO}_{3}$ film sputtered on a $\mathrm{Si}(100)$ substrate.

The MO transverse Kerr effect is related to a change in the reflectivity of the light irradiating a magnetic sample [3]. On the other hand, the PO effect is usually observed by ellipsometry and optical interferometry. In the present study, we use reflectometry to detect the PO effect. 


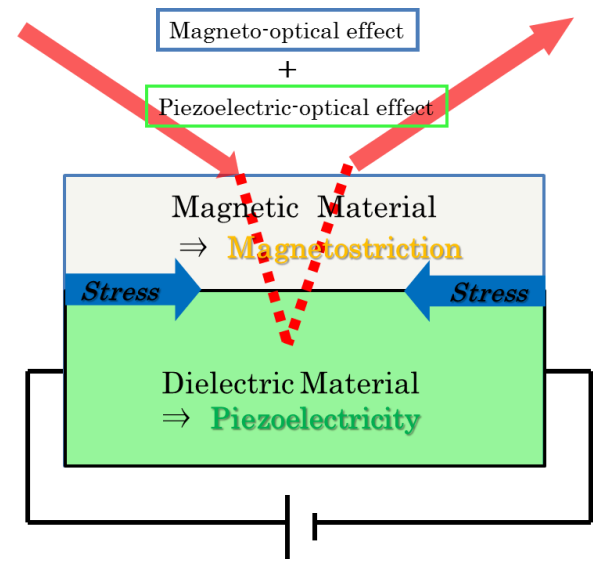

FIG. 1: Schematic diagram of magnetic/dielectric hybrid material and its optical response, the magneto-optical (MO) and the piezoelectric-optical (PO) effects, when an electric field is applied. Its optical response is affected by magnetostriction and the piezoelectricity of each layer.

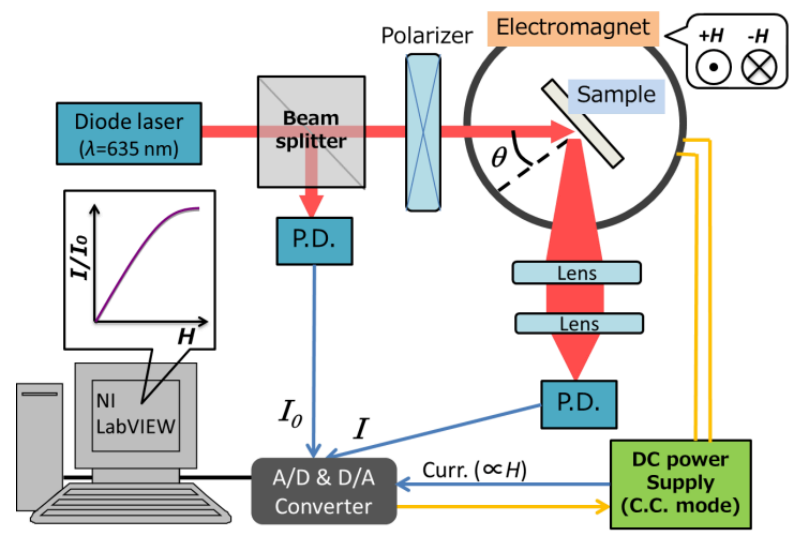

FIG. 2: Experimental set-up of homemade reflection optical measurement system for observing the MO Kerr effect.

\section{MEASUREMENT OF MAGNETO-OPTICAL KERR EFFECT IN A NICKEL FERRITE FILM}

Figure 2 illustrates the homemade optical measurement system used in this study. It can detect the MO effect. The light source is a diode laser (Thorlabs Ltd., CPS196; $\lambda=635 \mathrm{~nm}$ ) and the detector is a photodiode (Thorlabs, PDA-100A). The incident intensity is monitored using a beam split by a beam splitter (Thorlabs, CS1-BS1); for convenience, we here refer to it as the "reflectivity". The polarization of the incident beam is adjusted by a GlanThompson prism (Thorlabs, GTH5M). The change in the reflectivity on applying a magnetic field is measured. The current through the electromagnet (Tamagawa Co. Ltd., TM-WF44.208R-053) is controlled by a D/A converter (Nat. Inst. Co. Ltd., USB-6229). The reflection intensity and the monitored incident intensity are simultaneously detected by an A/D converter (NI, USB-9162).

It is not necessary to use an analyzer in this system because we are interested in the MO transverse Kerr effect. The magnetic field is applied normal to the scattering plane of the light. The magnitude of the transverse Kerr

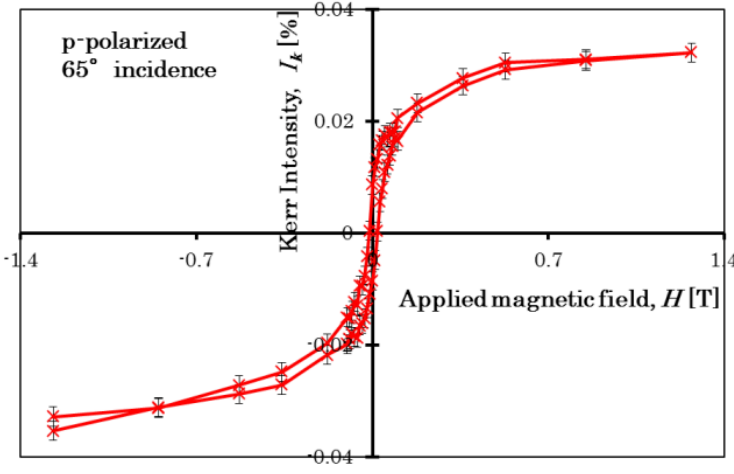

FIG. 3: Kerr hysteresis curve of sol-gel $\mathrm{NiFe}_{2} \mathrm{O}_{4} / / \mathrm{Si}$ for observing MO transverse Kerr effect. The magnetic layer is $2000 \mathrm{~nm}$ thick.

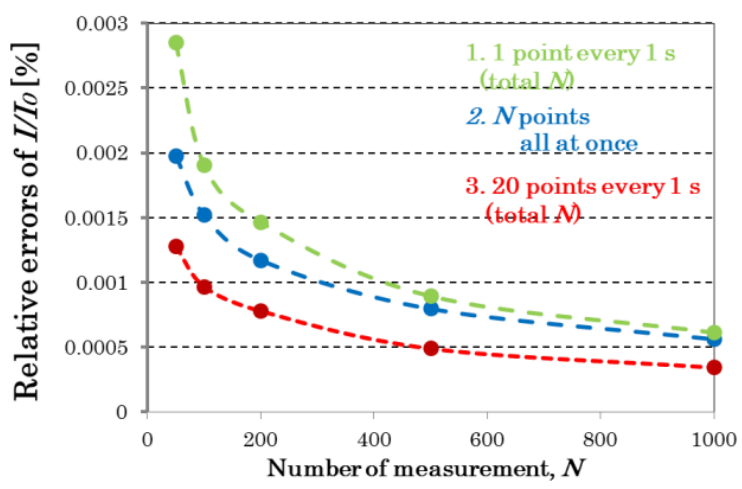

FIG. 4: Relative errors of normalized reflected beam intensity, $I / I_{0} . I_{0}$ is intensity of beam separated from an incident intensity by a beam splitter.

effect is defined by the Kerr intensity, which is given by

$$
\begin{aligned}
I_{\mathrm{K}} & =\Delta\left(I / I_{0}\right)_{H} \\
& =\left(\frac{\left(I / I_{0}\right)_{H}}{\left\{\left(I / I_{0}\right)_{H \rightarrow+0}+\left(I / I_{0}\right)_{H \rightarrow-0}\right\} / 2}-1\right) \times 100(\%)
\end{aligned}
$$

where $\left(I / I_{0}\right)_{H}$ is the reflectivity obtained on applying a magnetic field with strength $H$. The subscript $H \rightarrow+0$ denotes zero field when sweeping the field from positive to negative, while the subscript $H \rightarrow-0$ denotes zero field when the magnetic field is swept in the opposite direction. The rotation angle of polarization is not observed for the MO transverse Kerr effect.

Figure 3 shows the Kerr hysteresis curve obtained for a sol-gel $\mathrm{NiFe}_{2} \mathrm{O}_{4}$ film spin-coated on a silicon (100) single crystal. It is a plot of the Kerr intensity against the magnitude of the magnetic field. The Kerr intensity is related to the magnetization normal to the scattering plane of the light. The Kerr loop must exhibit a magnetic hysteresis curve. Therefore, the prepared $\mathrm{NiFe}_{2} \mathrm{O}_{4}$ has ferromagnetic and superparamagnetic phases.

The Kerr intensity saturated at a very low value of $0.03 \%$. The signal detection error was carefully reduced in the following manner. As mentioned above, the intensities of the reflected and monitored incident beams $(I$ and $I_{0}$, respectively) are simultaneously detected. The relative error in $I / I_{0}$ is calculated from the errors in $I$ 


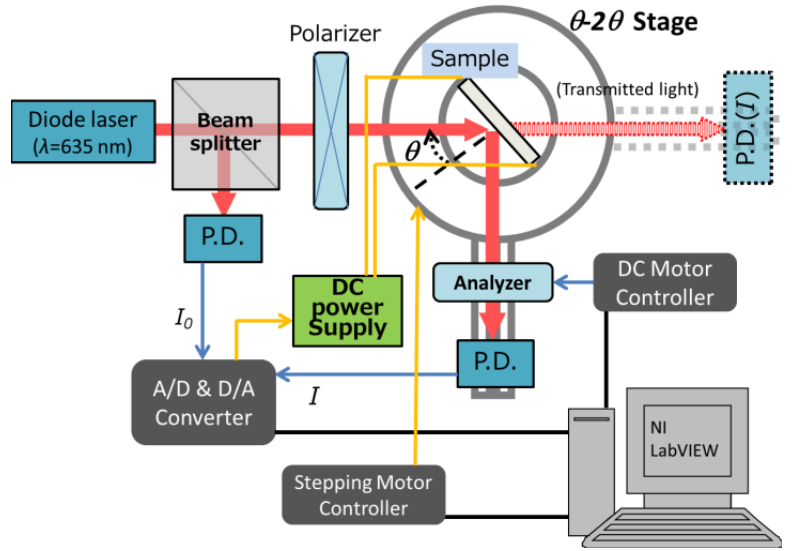

FIG. 5: Experimental set-up of a homemade optical measurement system for PO effect.

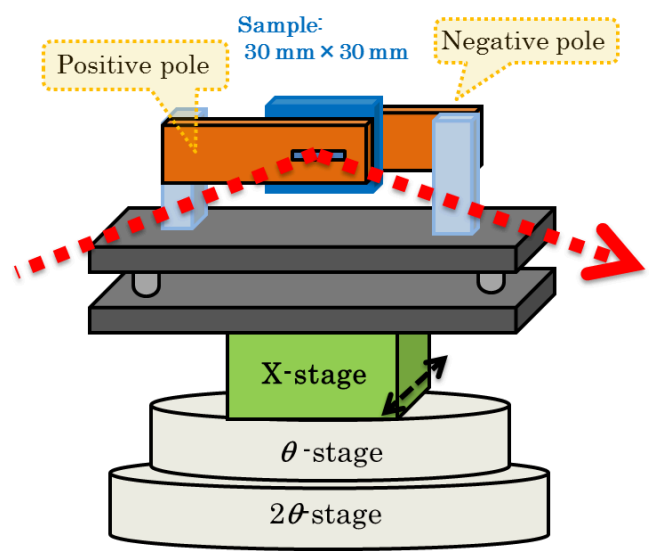

FIG. 6: Schematic diagram of sample holder equipped with system for applying an electric field.

and $I_{0}$. Figure 4 compares the relative errors in the beam intensity for three different detection schemes. The first method involves measuring one point per second for a total of $N$ times. The second method involves measuring $N$ points almost simultaneously. In our system, the relative error in the beam intensity is reduced such that 20 data points of the analog signal are transferred from the photodiode and are almost instantaneously detected by an A/D converter; this detection is repeated $N$ times every second. The last method is the best detection method because it minimizes the error.

\section{MEASUREMENT OF PO EFFECT FOR LITHIUM NIOBATE SINGLE CRYSTAL FOR TRANSMITTANCE AND REFLECTION}

We also want to detect the PO effect in a similar manner to the MO effect. Figure 5 shows the experimental set-up of the homemade optical system. It is equipped with a system for applying an electric field. Apart from this, the system is similar to that used to detect the MO effect shown in Fig. 2. It is also equipped with an automatically controllable $\theta-2 \theta$ stage and an analyzer sys-

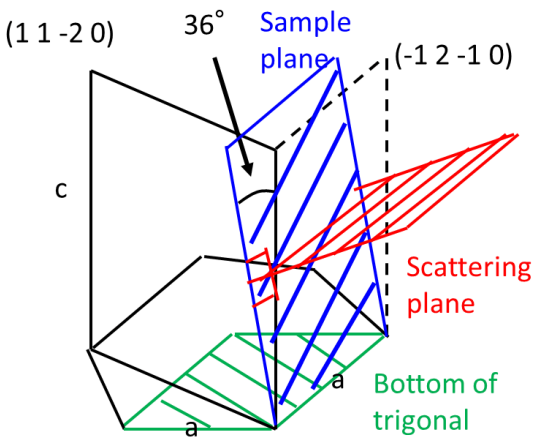

FIG. 7: Crystal orientation, scattering plane, and sample plane for $36^{\circ}$ plane of $\mathrm{LiNbO}_{3}$ trigonal single crystal.

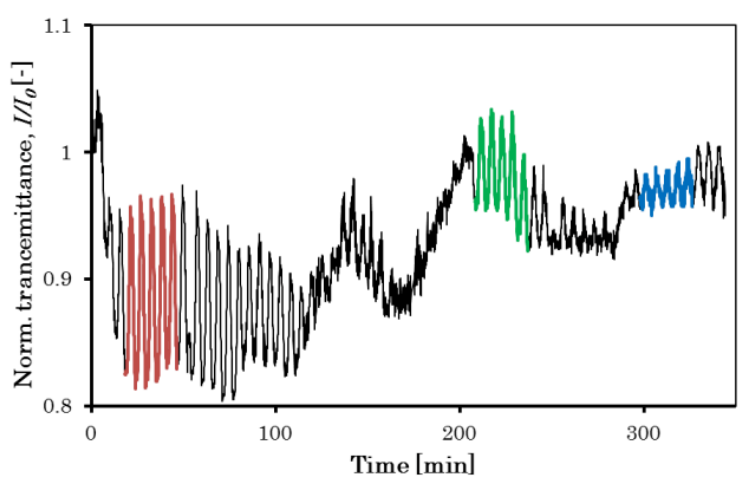

FIG. 8: Time series of normalized transmittance, $I / I_{0}$, during measurement of $\mathrm{PO}$ effect of $\mathrm{LiNbO}_{3}$ single crystal.

tem. Therefore, the transmittance, reflectivity, and polarization rotation can be automatically detected in both transmission and reflection modes. The sample is sandwiched between a pair of titanium plates, which function as electrodes (Fig. 6). The electric field is applied normal to the sample plane. The $x$-stage between the sample holder and the $\theta-2 \theta$ stage is used to adjust the position of the sample plane relative to the optical axis.

The lithium niobate $\left(\mathrm{LiNbO}_{3}\right)$ sample is a trigonal single crystal. As shown in Fig. 7, the sample plane is rotated $36^{\circ}$ relative to the $Y$-plane. This sample is utilized in a surface acoustic wave (SAW) device. For the trigo-

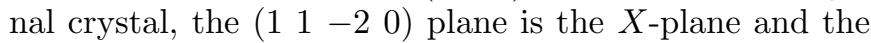
$\left(\begin{array}{lll}-1-2-1 & 0\end{array}\right)$ is the $Y$-plane. The $Z$-axis, $\left[\begin{array}{llll}0 & 0 & 0 & 1\end{array}\right]$, is the optical slow axis. The lattice constants $a$ and $c$ are 5.148 and $13.863 \AA$, respectively. The scattering plane (for reflection) and the light polarization (for reflection and transmission) lie in the red plane in Fig. 7.

The transmittance PO effect is observed for the first time in the present experiment. In a similar manner to the MO intensity, the PO intensity $I_{p}$ is defined as

$$
\begin{aligned}
I_{\mathrm{p}} & =\Delta\left(I / I_{0}\right)_{E} \\
& =\left(\frac{\left(I / I_{0}\right)_{E}}{\left\{\left(I / I_{0}\right)_{E \rightarrow+0}+\left(I / I_{0}\right)_{E \rightarrow-0}\right\} / 2}-1\right) \times 100(\%),
\end{aligned}
$$

where $\left(I / I_{0}\right)_{E}$ is the transmittance or the reflectivity when an electric field strength $E$ is applied. Figure 8 shows a time series of the transmittance measured by 


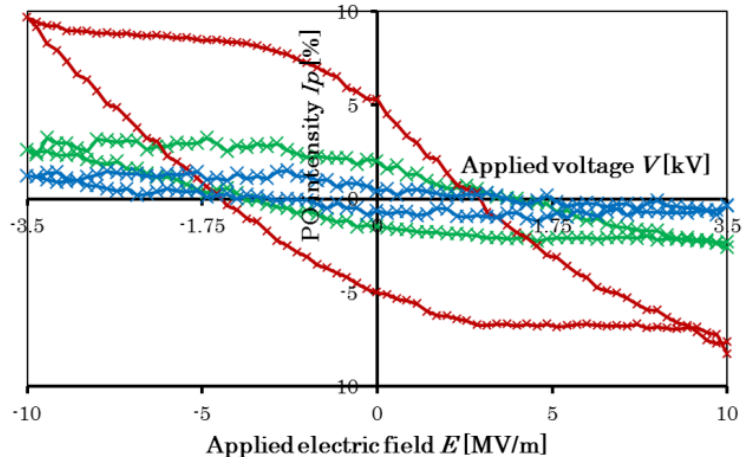

FIG. 9: Transmittance PO loops for red, green, and blue regions shown in Fig. 8.

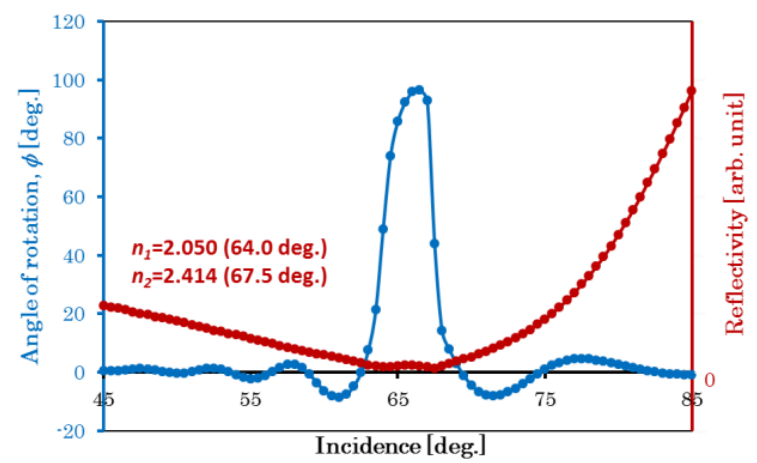

FIG. 10: Angle of rotation and reflectivity of $\mathrm{LiNbO}_{3}$ single crystal plotted against angle of incidence.

sweeping the electric field. The transmittance is normalized by the first point. The PO intensity exhibits short and long cycles. The long cycle corresponds to the variation in $E$. The short cycle was found to be independent of fluctuations in the beam intensity. In the red, green, and blue regions in Fig. 8, the PO intensity was averaged over several short cycles. The PO loop of each region is illustrated by the curves of the corresponding color in Fig. 9, where the PO intensity is plotted against the magnitude of the electric field. While the PO intensity differs in different regions, the curves exhibit similar shapes. Therefore, the short cycle of the PO intensity is probably due to the intrinsic PO effect. The long cycle is caused by changes in the sample position, which is affected by the inverse piezoelectric effect. It is very difficult to reduce the instability in the sample position, even by using our sample holder.

Since the goal of this study is to distinguish the PO and $\mathrm{MO}$ effects in the hybrid material, reflection is more suitable than transmission for detecting the optical response. Before measuring the reflection PO effect, we measured the angle of rotation and the reflectivity of the lithium niobate single crystal, as shown in Fig. 10. A birefringent material has two Brewster angles, which correspond to the refractive indices for the ordinary and extraordinary rays. The angle of rotation was a maximum at the Brewster angle.

The PO effect was observed by irradiating a $p$-polarized beam at an angle of incidence of $65^{\circ}$. Several PO loops

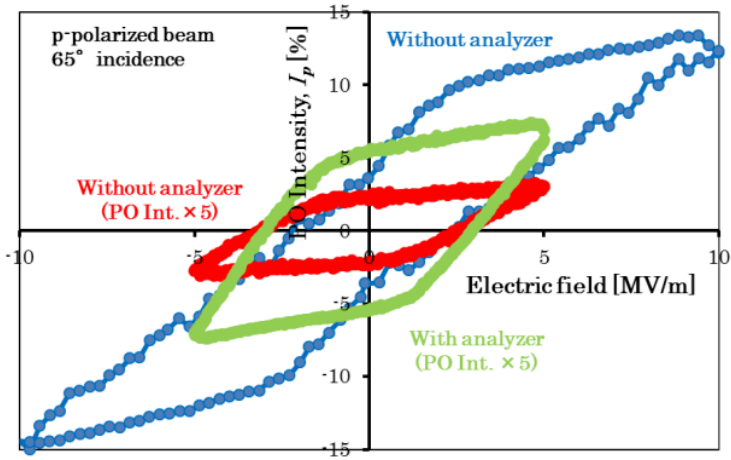

FIG. 11: Reflection PO loops with and without analyzer for $\mathrm{LiNbO}_{3}$ single crystal.

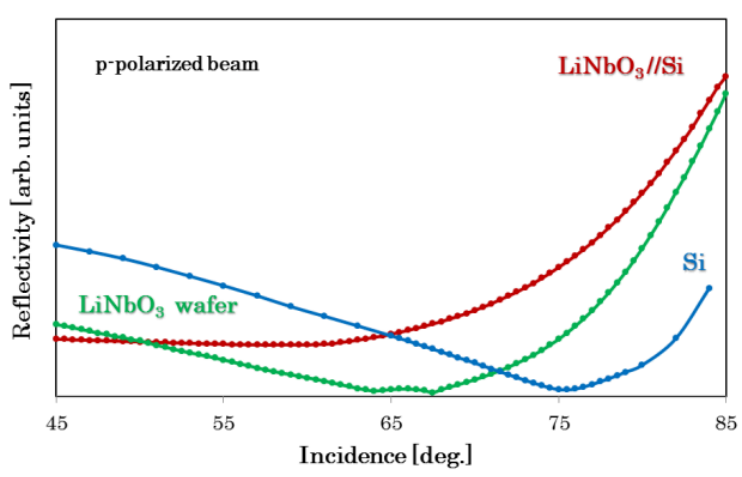

FIG. 12: Dependence of reflectivity on angle of incidence for 50-nm-thick sputtered $\mathrm{LiNbO}_{3}$ film together with reflectivities of $\mathrm{LiNbO}_{3}$ single crystal wafer and silicon single crystal wafer.

were obtained, as shown in Fig. 11. The red and green loops respectively indicate the PO loops observed without and with an analyzer, when a maximum magnitude of the electric field of $5 \mathrm{MV} / \mathrm{m}$ was applied. These loops have similar shapes; only the PO intensity differed. Both PO loops are consistent with the polarization behavior of ferroelectric materials; they are minor hysteresis loops in the absence on an electric field. For the red PO intensity loop in Fig. 11, the reflection intensity was detected without an analyzer. On the other hand, an analyzer was used to obtain the green loop; its azimuth was rotated by $45^{\circ}$ relative to the polarization of the reflection in zero field. Consequently, the data detected by the analyzer contains not only the angle of rotation but also the reflectivity. The PO intensity does not saturate even when an electric field of $10 \mathrm{MV} / \mathrm{m}$ is applied, as the blue loop shows. It is difficult to distinguish the electro-optical effect that originates from the dielectric polarization from the inverse piezoelectric effect due to the length of the material changing, for the same reason as for the transmission $\mathrm{PO}$ effect. 


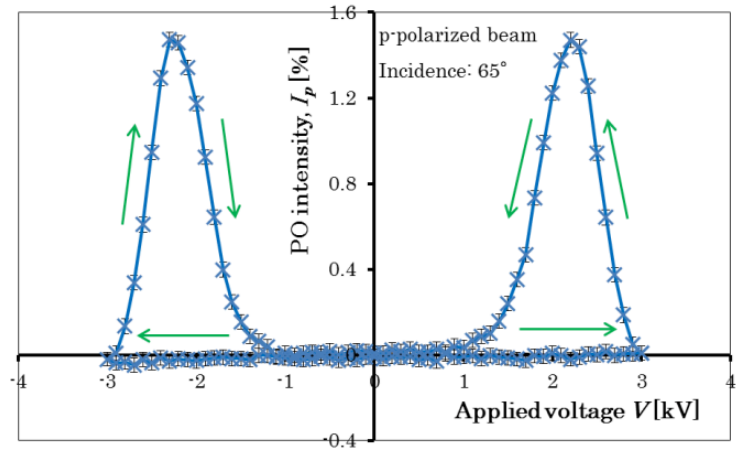

FIG. 13: Dependence of PO intensity on voltage applied to Si wafer, which was used as a substrate. Arrows indicate voltage sweeping direction.

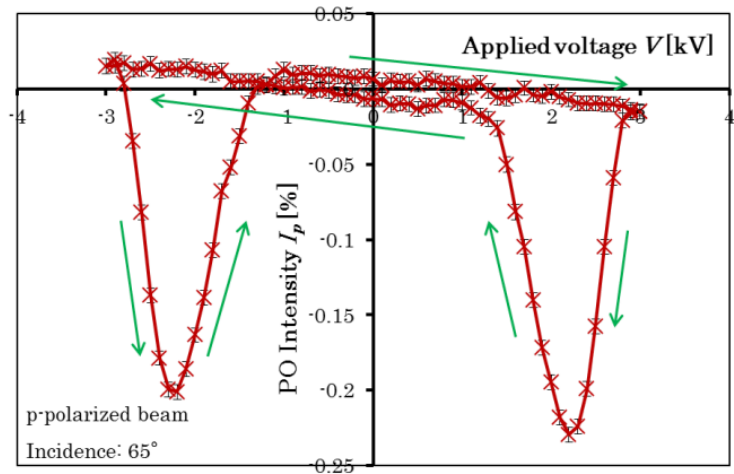

FIG. 14: Dependence of PO intensity on voltage applied to $\mathrm{LiNbO}_{3} / /$ Si sample.

\section{REFLECTION PIEZOELECTRIC-OPTICAL EFFECT FOR SPUTTERED LITHIUM NIOBATE FILM}

The inverse piezoelectric effect is expected to be reduced by using a thinner dielectric material. We sputtered a 50-nm-thick $\mathrm{LiNbO}_{3}$ film on a silicon (100) wafer. Figure 12 shows the reflectivity of the $\mathrm{LiNbO}_{3} / / \mathrm{Si}$ sample together with those of a $\mathrm{Si}$ wafer and a bulk $\mathrm{LiNbO}_{3}$ wafer. The Brewster angle was not clearly observed for the $\mathrm{LiNbO}_{3}$ film. $\mathrm{LiNbO}_{3} / / \mathrm{Si}$ exhibited a different reflectivity from the $\mathrm{LiNbO}_{3}$ wafer and the Si substrate.

The reflectivity of the $\mathrm{Si}$ wafer, which was used as a substrate, was observed by sweeping the magnitude of the electric field, $V$. The angle of incidence was selected to be $65^{\circ}$, which is close to the Brewster angle of $\mathrm{LiNbO}_{3}$. Figure 13 shows the change in the reflectivity, defined as the PO intensity by Eq. (2). The PO loop exhibited hysteresis. Peaks (1.5\%) in the positive intensity were observed for an electric field of $\pm 2.3 \mathrm{kV}$. The symmetric behavior relative to the magnitude of the applied voltage is probably due to the change in the dielectric constant, which is caused by the charged surface [4].

As shown in Fig. 14, the PO intensity of $\mathrm{LiNbO}_{3} / / \mathrm{Si}$ exhibits two peaks that are similar to those of the Si substrate except for the sign of the intensity. However, the peak intensity was asymmetric relative to the applied voltage. It is necessary to determine why the sign of the peak

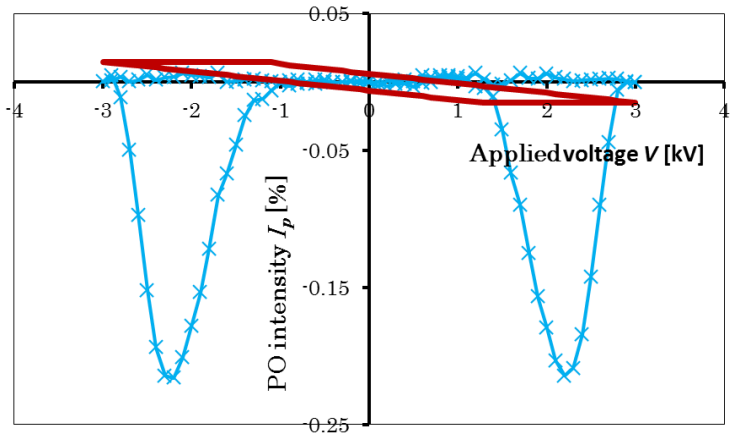

FIG. 15: Minor PO loop (red curve) and symmetric peak intensity (blue curve) separated by analysis of loop for $\mathrm{LiNbO}_{3} / / \mathrm{Si}$ sample shown in Fig. 14.

intensity for this sample is reversed and why the absolute intensity is much lower than that for Si.

The sign of the peak intensity is thought to be due to multiple scattering or the birefringence of the dielectric layer. However, the peak is not due to a change in the dielectric constant of the Si substrate; rather, it is due to a change in the dielectric constant of the $\mathrm{LiNbO}_{3}$ film layer.

The hysteresis in the PO intensity is separate from the peaks. A numerical analysis of the PO loop shown in Fig. 14 enabled the loop to be divided into two hysteresis curves, as shown in Fig. 15. The blue loop is symmetric relative to the applied field and it is the similar to the loop of the Si substrate except for the intensity and the sign of the peak. The red loop is separate from the blue loop and is similar to the minor dielectric PO loop in Figs. 9 and 11. Hence, the sputtered $\mathrm{LiNbO}_{3}$ film layer is estimated to have a net saturated PO intensity of $\pm 0.015 \%$.

For the measured PO loops in Figs. 13 and 14, the repeatability of the signal intensity is much better than that of the $\mathrm{LiNbO}_{3}$ wafer. This is due to the stability of the sample position resulting from the small inverse piezoelectric effect. However, the sample set still exhibited poor reproducibility.

\section{CONCLUSIONS}

We constructed optical measurement systems equipped with high sensitivity detectors to detect the $\mathrm{MO}$ and $\mathrm{PO}$ effects. These effects have signal intensities of the order of $0.01 \%$. The transverse Kerr intensity of a $\mathrm{NiFe}_{2} \mathrm{O}_{4}$ film and the $\mathrm{PO}$ intensity of a $\mathrm{LiNbO}_{3}$ film saturated at 0.03 and $0.015 \%$. In the future, we intend to separate these two effects for hybrid materials that have dielectric and magnetic phases by adjusting the optical conditions. Specifically, the charge stored on the sample surface will be reduced by applying an $\mathrm{AC}$ voltage so that time-resolved measurements will be effective for a higher-frequency field.

\section{Acknowledgments}

This work was supported by a Grant-in-Aid for Scientific Research (B, 20310079) from the Japan Society for 
the Promotion of Science (JSPS). A part of this work was conducted at the Nano-Processing Facility, supported by the IBEC Innovation Platform, AIST.

(1997).

[1] R. Ramesh and N. A. Spaldin, Nat. Mater. 6, 21 (2007).

[2] E. A. Eliseev, A. N. Morozovska, et al., Phys. Rev. B 82, 085408 (2010).

[3] K .Maruyama, K.Namikawa, et al., J. Appl. Phys. 81, 5675
[4] R. S. Jacobsen, K. N. Andersen, et al., Nature 114, 199 (2006). 